\title{
Nanoparticulate Mineralized Collagen Scaffolds Induce In Vivo Bone Regeneration Independent of Progenitor Cell Loading or Exogenous Growth Factor Stimulation
}

Xiaoyan Ren, MD, $\mathrm{PhD}^{1,2,3}$, Victor Tu, BS ${ }^{1,2,3}$, David Bischoff, $\mathrm{PhD}^{3}$, Daniel W. Weisgerber, $\mathrm{BA}^{4}$, Michael S. Lewis, MD, $\mathrm{PhD}^{5}$, Dean T. Yamaguchi, MD, $\mathrm{PhD}^{3}$, Timothy A. Miller, $\mathrm{MD}^{1,2}$, Brendan A.C. Harley, $\mathrm{ScD}^{4}$, Justine C. Lee, MD, $\mathrm{PhD}^{1,2,3}$

${ }^{1}$ Division of Plastic and Reconstructive Surgery, UCLA David Geffen School of Medicine, Los Angeles, CA 90095, ${ }^{2}$ Division of Plastic and Reconstructive Surgery, Greater Los Angeles VA Healthcare System, Los Angeles, CA 90073, ${ }^{3}$ Research Service, Greater Los Angeles VA Healthcare System, Los Angeles, CA $90073{ }^{4}$ Department of Chemical and Biomolecular Engineering, Institute for Genomic Biology, University of Illinois at

Urbana-Champaign, Urbana, IL 61801, ${ }^{5}$ Department of Pathology, Greater Los Angeles VA Healthcare System, Los Angeles, CA 90073

Keywords: bone regeneration, biomimetic material, nanoparticulate mineralization 


\section{Abstract}

Current strategies for skeletal regeneration often require co-delivery of scaffold technologies, growth factors, and cellular material. However, isolation and expansion of stem cells can be time consuming, costly, and requires an additional procedure for harvest. Further, the introduction of supraphysiologic doses of growth factors may result in untoward clinical side effects, warranting pursuit of alternative methods for stimulating osteogenesis. In this work, we describe a nanoparticulate mineralized collagen glycosaminoglycan scaffold that induces healing of critical-sized rabbit cranial defects without addition of expanded stem cells or exogenous growth factors. We demonstrate that the mechanism of osteogenic induction corresponds to an increase in canonical BMP receptor signalling secondary to autogenous production of BMP-2 and -9 early and BMP-4 later during differentiation. Thus, nanoparticulate mineralized collagen glycosaminoglycan scaffolds may provide a novel growth factor-free and ex vivo progenitor cell culture-free implantable method for bone regeneration. 


\section{Introduction}

Skeletal regenerative medicine emerged as a field of investigation to address the clinical need for reconstruction of osseous deficiencies secondary to congenital, traumatic, and post-oncologic conditions. Although reconstructive surgical techniques have undergone significant advances, correction still depends on the availability of tissue within the patient and the morbidity that a patient can sustain from tissue transfer $[1,2]$. Non-autologous replacement in the form of allograft and alloplast are fraught with problems including resorption, exposure, and infection [3-5]. These limitations demonstrate a significant need for establishing alternative methods for bone replacement.

Approaches to bone tissue engineering incorporate three elements: osteogenic cells, growth factors, and scaffolding material $[6,7]$. Cell types are usually adult tissuederived stem cells and osteogenic growth factors are frequently members of the bone morphogenetic protein (BMP) family [8]. Mechanistically, BMP dimers promote osteogenic differentiation by binding to BMP receptor (BMPR) complexes and activating intracellular signaling cascades in osteoprogenitor cells [9]. Depending on the method of BMPR oligomerization, activation of the canonical or non-canonical pathways may occur. In the canonical pathway, the receptor Smads (Smad 1/5/8) are recruited and phosphorylated. Phosphorylated receptor Smads associate with co-Smad (Smad 4) and translocate to the nucleus to activate transcription. In the non-canonical pathway, ERK, p38 MAPK, and PI3K/Akt are activated. Regulation of each BMP receptor signaling may occur at the ligand level, receptor level, or via intracellular molecules [10-12]. Secreted homodimeric BMP antagonists such as noggin, chordin, and the DAN/Cerberus family of 
proteins sequester BMP ligands $[13,14]$. The pseudoreceptor BMP and activin bound protein (BAMBI) inhibits the effects of the activated receptors and is expressed in osteoblasts [15]. Intracellular negative or positive regulation can also occur within or between the canonical and noncanonical pathways with expression of inhibitory Smad proteins.

Clinically, the Food and Drug Administration (FDA) has approved two BMPs for usage in bone defects: BMP-2 and -7 [16-18]. However, complications such as soft tissue swelling, ectopic bone formation, resorption of adjacent bone, and long term effects on maxillary growth have all been reported [19-21]. Such drawbacks highlight the need for alternative methods for augmenting osteogenesis without reliance on supraphysiologic dosages of growth factors.

Scaffolding material, once considered to be inert or passive supporters of biological processes, are increasingly designed to have instructive properties that promote osteogenesis depending on the material, porosity, and ability to mimic the native extracellular matrix (ECM) [6]. Although a multitude of scaffolds have been reported in the literature comprised of ceramic, biodegradable polymers, extracellular matrix components, or combinations thereof, the quest for the ideal scaffold is yet to be complete. Classic limitations include production of inflammatory acid metabolites by biodegradable polymers and structural contraction of collagen scaffolds [22-24]. Although ceramic composites, such as $\beta$-tricalcium phosphate ( $\beta$-TCP) and hydroxyapatite, are both osteoconductive and osteoinductive, their utility is limited by variable resorption rates or brittle mechanical properties [25]. We recently reported that combining both the organic and inorganic components of the ECM in the form of a novel nanoparticulate mineralized collagen glycosaminoglycan (MC-GAG) scaffold results in a 
highly osteogenic and structurally stable scaffold that stimulates osteogenic differentiation of human mesenchymal stem cells (hMSCs) by autogenously stimulating the canonical BMPR signaling pathway $[26,27]$. In this work, we evaluate the therapeutic potential of MC-GAG in bone regeneration by investigating in vivo calvarial bone healing in a rabbit cranial defect model.

\section{Materials and Methods}

\section{Fabrication of non-mineralized and mineralized collagen scaffolds}

Col-GAG and MC-GAG scaffolds were prepared using the lyophilization process described previously [28]. Briefly, a suspension of collagen and GAGs were produced by combining microfibrillar, type I collagen (Collagen Matrix, Oakland, NJ) and chondroitin-6-sulfate (Sigma-Aldrich, St. Louis, MO) with and without calcium salts (calcium nitrate hydrate: $\mathrm{Ca}\left(\mathrm{NO}_{3}\right)_{2} \cdot 4 \mathrm{H}_{2} \mathrm{O}$; calcium hydroxide: $\mathrm{Ca}(\mathrm{OH})_{2}$, Sigma-Aldrich,

St. Louis, MO) in a solution of acetic acid (Col-GAG) or phosphoric acid (MC-GAG). The suspension was frozen using a constant cooling rate technique $\left(1^{\circ} \mathrm{C} / \mathrm{min}\right)$ from room temperature to a final freezing temperature of $-10^{\circ} \mathrm{C}$ using a freeze dryer (Genesis, VirTis) and sublimated under vacuum $\left(<200 \mathrm{mTorr}, 0^{\circ} \mathrm{C}\right)$. Scaffolds were crosslinked with 1-ethyl-3-(3-dimethylaminopropyl) carbodiimide (EDAC, Sigma Aldrich) and Nhydroxysuccinimide (NHS, Sigma Aldrich) in distilled, deionized water for $2 \mathrm{~h}$ at room temperature and washed with PBS [29]. The height of the scaffold specimens used in this study were $5.8 \pm 0.4 \mathrm{~mm}$. Scaffold porosity was $85 \pm 3 \%$ [30], pore size was $156 \pm$ $6 \mu \mathrm{m}[30,31]$, and morphology consisted of isotropic pores with a transverse:longitudinal pore aspect ratio of $0.95 \pm 0.01$ [31] as we previously reported. Disks $8 \mathrm{~mm}$ in 
diameter were prepared for in vitro studies using punch biopsies. Disks $14 \mathrm{~mm}$ in diameter were prepared for in vivo studies.

\section{Animals and Cell culture}

Isolation of rabbit bone marrow stromal cells and cell culture for in vitro studies:

New Zealand White rabbits (2-3 months old, cared for in compliance with the USDA Animal Welfare Act and PHS Policy for the Humane Care and Use of Laboratory Animals) were injected with $3000 \mathrm{U}$ heparin and $390 \mathrm{mg}$ of pentobarbital solution (FatalPlus $^{\circledR}, 390 \mathrm{mg} / \mathrm{ml}$ ). Bone marrow was obtained from long bones via aspiration and resuspended in PBS by successive passages through 18 and 20 gauge syringe needles. Cells were cultured in $\alpha$-MEM (Cellgro Mediatech, Manassas, VA) containing $10 \%$ fetal bovine serum (FBS) (Atlanta Biologicals, Flowery Branch, GA), 2 mM L-glutamine (Fisher Scientific, Waltham, MA), and $100 \mathrm{IU} / \mathrm{mL}$ penicillin/100 $\mu \mathrm{g} / \mathrm{mL}$ streptomycin (Fisher Scientific) at $37^{\circ} \mathrm{C}$ for 2 days. Adherent cells were identified as stromal cells. At passage 3, $3 \times 10^{5}$ BMSCs were seeded onto 8-mm Col-GAG and MC-GAG scaffolds in proliferation media. 24 hours after seeding, proliferation media was exchanged for osteogenic differentiation media consisting of $10 \mathrm{mM} \beta$-glycerophosphate, $50 \mu \mathrm{g} / \mathrm{ml}$ ascorbic acid and $0.1 \mu \mathrm{M}$ dexamethasone. Scaffolds were untreated or treated with rhBMP-2 at a concentration of $50 \mathrm{ng} / \mathrm{mL}$. Media and BMP-2 were changed every 2-3 days during culture.

In vivo rabbit cranial defect reconstruction: Approximately three weeks prior to creation of the parietal defect, 26 female New Zealand White rabbits (2-3 months old) underwent bone marrow harvest from the ilium as described above. Preoperatively, rabbits were injected subcutaneously with enrofloxacin $(5 \mathrm{mg} / \mathrm{kg})$ and acepromazine (1 
$\mathrm{mg} / \mathrm{kg}$ ). Anesthesia was maintained with isoflurane gas (1.5-3\%) during the procedure and pain control was performed with buprenorphine $0.05 \mathrm{mg} / \mathrm{kg}$ and carprofen $4 \mathrm{mg} / \mathrm{kg}$ subcutaneous injection. Isolated BMSCs were cultured as described above and $2 \times 10^{6}$ cells were seeded onto 14-mm Col-GAG and MC-GAG scaffolds in proliferation media. 24 hours after seeding, proliferation media was exchanged for osteogenic differentiation media consisting of $10 \mathrm{mM} \beta$-glycerophosphate, $50 \mu \mathrm{g} / \mathrm{ml}$ ascorbic acid and $0.1 \mu \mathrm{M}$ dexamethasone. Scaffolds were untreated or treated with rhBMP-2 at a concentration of $50 \mathrm{ng} / \mathrm{mL}$ for one week. Media and BMP-2 were changed every 2-3 days.

The rabbits were divided into seven groups (3 - 4 rabbits per group): 1) defect without reconstruction, 2) Col-GAG scaffold only, 3) Col-GAG seeded with BMSCs ex vivo, 4) Col-GAG seeded with BMSCs and rhBMP-2 ex vivo, 5) MC-GAG scaffold only, 6) MC-GAG seeded with BMSCs ex vivo, 7) MC-GAG seeded with BMSCs and rh-BMP2 ex vivo. The head of each rabbit was shaved and disinfected with Betadine. The cranial surface was exposed by a midline incision and the overlying parietal periosteum was dissected off of the calvarium. For each rabbit, a $14 \mathrm{~mm}$ full thickness, extradural defect was created by a hand powered trephine and the bone was lifted away without injury to the dura [32]. One scaffolds was implanted for each rabbit and the incision was closed with 4-0 nylon sutures.

Twelve weeks after implantation, the rabbits were euthanized by intravenous injection of $1 \mathrm{~mL}$ of pentobarbital solution (Fatal-Plus ${ }^{\circledR}, 390 \mathrm{mg} / \mathrm{ml}$ ) intravenously via the marginal ear vein. The previous incision was then reopened and the calvarium was exposed. The calvarium including the cranial defect was analyzed grossly and then explanted for micro-CT, histologic, and biomechanical analyses. 


\section{Quantitative real-time reverse-transcriptase polymerase chain reaction for in vitro studies}

Scaffolds were processed for total RNA extraction using the RNeasy kit (Qiagen, Valencia, CA) at indicated timepoints including $0,3,7,14$, and 24 days of culture. Quantitative real-time reverse-transcriptase polymerase chain reaction (RT-PCR) was performed on the Opticon Continuous Fluorescence System (Bio-Rad Laboratories, Inc., Hercules, CA) using the QuantiTect SYBR Green RT-PCR kit (Qiagen) and cycle conditions described previously [26]. Cycle conditions were as follows: reverse transcription at $50^{\circ} \mathrm{C}$ (30 minutes); activation of HotStarTaq DNA polymerase/inactivation of reverse transcriptase at $95^{\circ} \mathrm{C}$ ( 15 minutes); and 45 cycles of $94^{\circ} \mathrm{C}$ for 15 seconds, $58^{\circ} \mathrm{C}$ for 30 seconds, and $72^{\circ} \mathrm{C}$ for 45 seconds. Results were analyzed using the comparative CT method for analyzing reverse-transcriptase polymerase chain reaction data and presented as representative graphs of triplicate experiments. Data was expressed as a fold change from day 0 levels. Primers used are listed with accession numbers in Table 1.

\section{Histology and immunohistochemistry}

In vitro studies: Scaffolds were fixed at $10 \%$ normal buffered formalin, embedded in paraffin, and sectioned at 4 microns using standard techniques. The sections were deparaffinized and stained with hematoxylin and eosin, Alizarin Red, or anti-p-Smad1/5 (Cell Signaling Techologies, Beverly, MA) and processed with the Dako automated FLEX system (Dako, Carpinteria, CA). All slides were analyzed qualitatively using a standard microscope and digitally photographed. 
In vivo cranial defects: Skulls were removed twelve weeks after the implantation of scaffolds ( $n=3-4$ skulls per group), fixed in 10\% formalin, embedded in paraffin, and sectioned at 4 microns using standard techniques. The sections were deparaffinized and stained with hematoxylin and eosin. All slides were analyzed qualitatively using a standard microscope and digitally photographed.

\section{Micro-Computed Tomographic Imaging}

Micro-computed tomographic imaging $(\mu \mathrm{CT})$ was perfomed using the Scanco $\mu \mathrm{CT} 35$ (Scanco Medical AG, Bruttisellen, Switzerland).

In vitro studies: Mineralization was followed by $\mu \mathrm{CT}$ after 6 and 12 weeks in culture ( $n$ $=3$ scaffolds per time point). Scaffolds were fixed using $10 \%$ formalin for 24 hours and then stored in $70 \%$ ethanol at $4^{\circ} \mathrm{C}$ until scanned. Prior to scanning, the scaffolds were wetted in PBS and then placed in the holder (without solution) and scanned quickly (15$30 \mathrm{~min}$ ) to prevent drying. Scans were performed using medium resolution settings with a source voltage of $70 \mathrm{E}(\mathrm{kVp})$, Intensity $(\mu \mathrm{A})$ of 114 , and a voxel size of $37 \mu \mathrm{m}$.

Scaffold areas were contoured to establish volumes of interest by visual examination of serial slices in all of the specimens. Two-dimensional images were analyzed using software supplied from Scanco (Image Processing Language version 5.6) to produce three-dimensional reconstruction pictures and volume determination. Optimum arbitrary threshold values of 20 (showing scaffold and mineralization) and 80 (mineralization alone) were used uniformly for all specimens to quantify mineralized areas from surrounding unmineralized scaffold.

In vivo cranial defects: Skulls were removed twelve weeks after the implantation of scaffolds ( $n=3-4$ skulls per group), fixed in 10\% formalin for 24 hours, and then stored 
in $70 \%$ ethanol at $4^{\circ} \mathrm{C}$ until scanned. Scans were performed in PBS using high resolution settings with a source voltage of $45 E(k V p)$, Intensity $(\mu A)$ of 177 , and a voxel size of $15 \mu \mathrm{m}$. Skull areas were contoured to establish volumes of interest and an optimum arbitrary threshold value of 415 was used uniformly for all specimens to quantify mineralized bone areas from surrounding unmineralized scaffold. Threedimensional reconstruction pictures were generated and the volume of old and new bone calculated.

For density calculations, DICOM files were imported into Osirix (Pixmeo Sarl) and mean Hounsfield Units (HU) were obtained from a cylindrical volume of $0.045 \mathrm{~cm}^{3}$ consisting of the defect and $0.03 \mathrm{~cm}^{3}$ outside of the defect in the native bone as an internal control. The ratio of mean density of defect/mean density of native bone was then calculated for each condition (defect only, empty Col-GAG, empty MC-GAG, ColGAG precultured with BMSCs, MC-GAG precultured with BMSCs, Col-GAG precultured with BMSCs and $50 \mathrm{ng} / \mathrm{mL}$ rhBMP-2, and MC-GAG precultured with BMSCs and 50 $\mathrm{ng} / \mathrm{mL}$ rhBMP-2).

\section{Western blot for In vitro studies}

Lysates for western blot analysis were prepared from scaffolds at $0,3,7,14$, and 24 days of culture using Phosphosafe lysis buffer (Novagen, Madison, WI). Equal amounts of protein lysates were subjected to $4-20 \%$ (Bio-Rad) SDS-PAGE. Western analysis was carried out with antibodies against phosphorylated-Smad1/5 (p-Smad1/5), total Smad1/5/8, phosphorylated-ERK1/2 (p-ERK1/2), total ERK1/2, phosphorylated-Smad2/3 (p-Smad2/3), total Smad2/3, phosphorylated-p38 (p-p38), total p38, Akt, phosphorylated-Akt ( $\mathrm{p}$-Akt) and $\beta$-actin followed by 1:4000 dilutions of horseradish peroxidase-conjugated IgG antibodies (Bio-Rad, Hercules, CA) and an enhanced 
chemiluminescent substrate (Thermo Scientific, Rockford, IL). For detection of pSmad1/5 and total Smad1/5/8, $40 \mu \mathrm{g}$ of lysate was loaded per lane. For detection of $p$ ERK1/2 and total ERK1/2, $60 \mu \mathrm{g}$ of lysate was loaded per lane. For detection p-p38, total p38, p-Smad2/3, total Smad2/3, $50 \mu \mathrm{g}$ of lysate was loaded per lane. All primary phospho antibodies were obtained from Cell Signaling Technologies (Beverly, MA) and all primary full length antibodies were obtained from Santa Cruz Biotechnology (Santa Cruz, CA). Imaging was carried out using ImageJ (NIH, Bethesda, MD).

\section{Reference point indentation for in vivo rabbit cranial defects}

Explanted rabbit skulls in $10 \%$ formalin were tested with the BioDent reference point indentation device (Active Life Scientific, Santa Barbara, CA) according to manufacturer's instructions. Indentations were conducted in five areas at a force of $2 \mathrm{~N}$, an indentation frequency of $2 \mathrm{~Hz}$, and 10 indentation cycles at a touchdown force of 0.1 $\mathrm{N}$ using a probe assembly type BP2. Indentation data was analyzed with the BioDent software for the total indentation distance (TID), first cycle indentation distance (ID1st), loading slope (LS), and unloading slopes (US). Toughness, or resistance to fracture, was determined by the total distance of indentation (TID) reached by the test probe and the first cycle indentation distance (ID1st). Relative stiffness was determined by the loading (LS) and unloading (US) slopes of the force (N) to displacement $(\mu \mathrm{m})$ curves. To minimize differences in the thickness of bone for each animal as well as the bone healing capabilities, data from each cranial defect was internally controlled with the native calvarial bone.

\section{Statistical Analysis}

All comparisons were performed with univariate analyses of variance (ANOVA) with post hoc comparisons using the Tukey criterion with SPSS software Version 23 (SPSS, Inc., 
Chicago, IL). Data points were composed of at least three independent experiments, unless otherwise indicated. The F statistic, associated degrees of freedom, and $p$ values are reported. For post hoc comparisons between groups using the Tukey criterion, $\mathrm{p}$ values are reported. A value of $p<0.05$ was considered significant. 


\section{Results}

\section{Mineralized collagen glycosaminoglycan scaffolds upregulate in vitro osteogenic gene expression and mineralization in rabbit bone marrow stromal cells.}

We recently reported that addition of mineral content via nanoparticulate calcium phosphate (CaP) to collagen glycosaminoglycan (Col-GAG) scaffolds significantly promoted osteogenesis of human bone marrow-derived mesenchymal stem cells [26, 27, 33, 34]. We also determined that hMSC osteogenic differentiation and mineralization occurred in a growth factor independent manner [26]. To test this effect on in vivo bone regeneration, we first determined whether this effect could be replicated in a rabbit model.

Col-GAG and mineralized collagen-GAG (MC-GAG) scaffolds were seeded with primary rabbit BMSCs isolated from lower extremity long bones and untreated or treated with rhBMP-2 $(50 \mathrm{ng} / \mathrm{mL})$, a concentration previously demonstrated in our laboratory to stimulate osteogenic differentiation in rabbit BMSCs [35]. Three transcriptional markers were chosen for evaluation of early and late phases of osteogenic differentiation: alkaline phosphatase (ALP), collagen I (Col I), and osteocalcin (OCN) (Figure 1A-C). A significant effect of type, timing, and treatment of scaffolds on gene expression was seen in ALP $[F(7,18)=5.694, p=0.001]$, Col I $[F(11,23)=12.217, p<0.001]$, and OCN $[F(11,22)=9.227, p<0.001]$. As an early marker of osteogenesis, post hoc comparisons indicated that ALP expression increased with BMP-2 treatment at day 3 for BMSCs cultured on Col-GAG $(p<0.05)$ or MC-GAG $(p<0.01)$ in comparison to untreated cultures. There were no statistically significant differences in ALP expression between the scaffolds. By day 7, ALP expression had increased in both scaffold types and BMP-2 
A

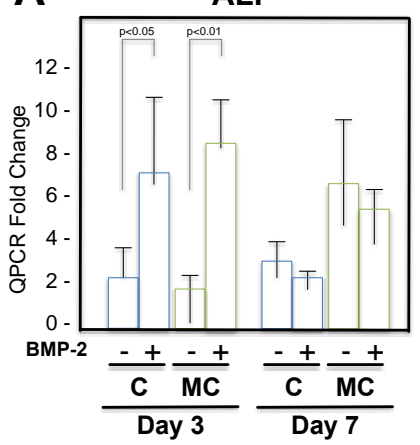

B

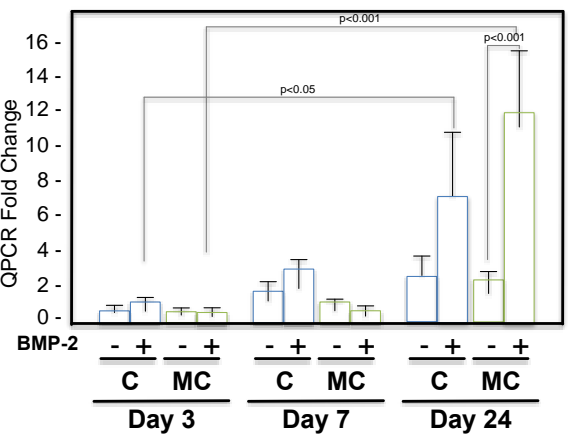

C

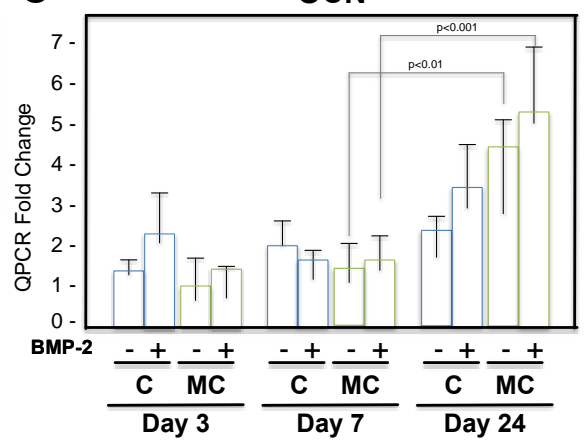

D

6 weeks

8 weeks

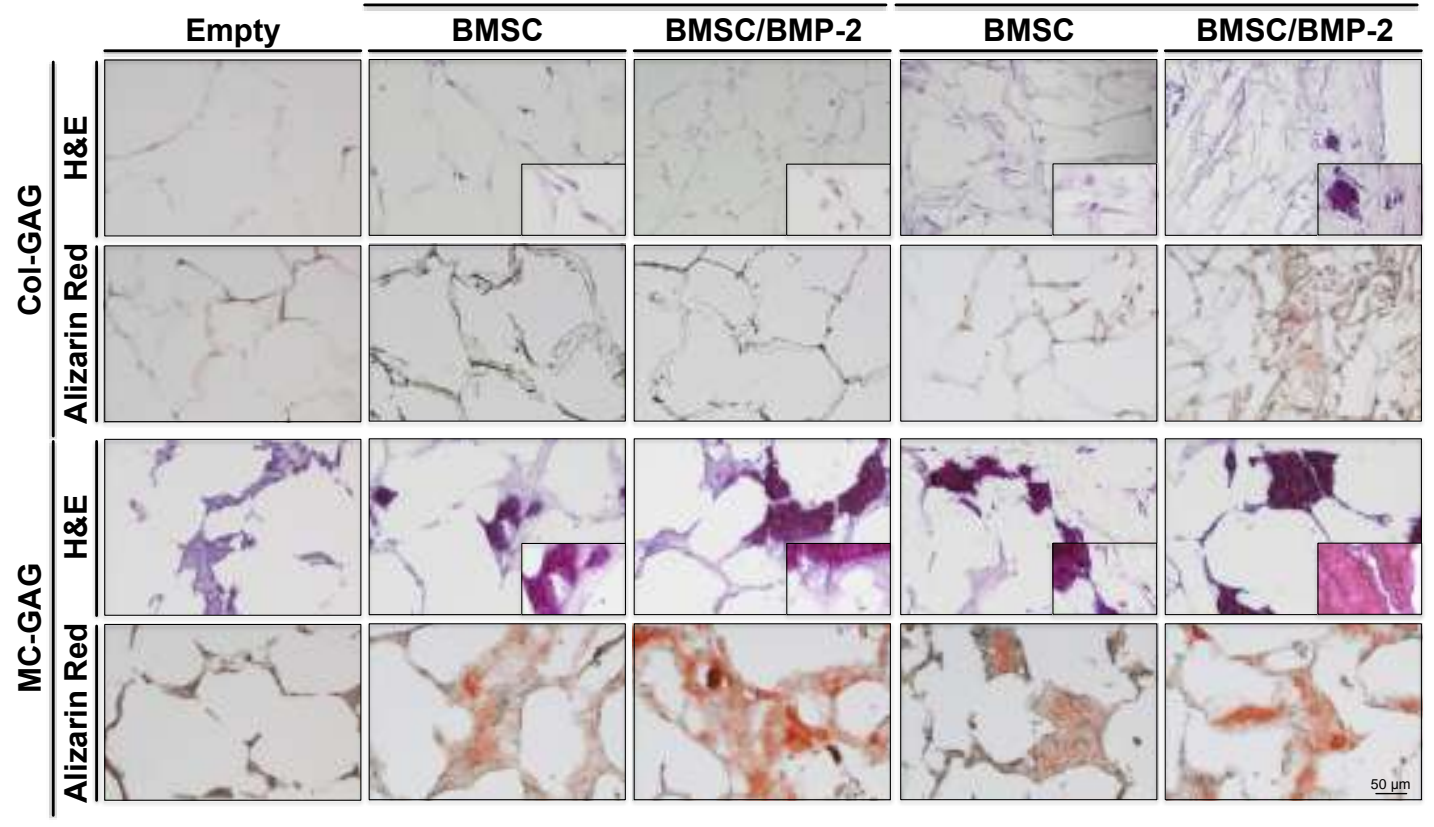

Figure 1. In vitro osteogenic gene expression and mineralization of BMSCs in response to BMP-2 on Col-GAG and MC-GAG scaffolds in the absence of presence of BMP-2. Real-time RT-PCR analysis of (A) alkaline phosphatase (ALP), (B) collagen I (Col I), and (C) osteocalcin (OCN) expression of BMSCs with and without BMP-2 $(50 \mathrm{ng} / \mathrm{mL})$ treatment in ColGAG and MC-GAG scaffolds. Data is expressed as the mean $\pm S D$ of three independent experiments in relation to day 0 values. $P$ values are shown in the figure. (D) H\&E and Alizarin Red staining of histologic sections of Col-GAG and MC-GAG scaffolds cultured with BMSCs in osteogenic medium in the presence or absence of BMP-2 $(50 \mathrm{ng} / \mathrm{mL})$ for 6 or 8 weeks. Insert in H\&E panels show higher magnification demonstrating visualization of cells or mineralized material. Empty scaffolds shown as control.

treatment no longer demonstrated any further increases.

As later markers for osteogenic differentiation, Col I and OCN were examined.

Unlike ALP, Col I transcripts did not demonstrate appreciable increases until day 24 of 
culture for either Col-GAG $(p<0.05)$ or MC-GAG $(p<0.001)$. Similar to ALP, these increases were sensitive to BMP-2 stimulation in either scaffold. Similar to Col I, OCN also demonstrated a delay prior to increase. At day $24, \mathrm{OCN}$ transcripts were found to be increased in MC-GAG scaffolds when compared to earlier timepoints in the absence $(p<0.01)$ or presence $(p<0.001)$ of BMP-2. Unlike Col I, OCN transcripts were not significantly affected by BMP-2 treatment. In contrast, OCN transcripts in Col-GAG scaffolds trended towards an increase in later timepoints but this did not achieve statistical significance.

To investigate whether the increases in osteogenic gene expression translated to increased mineralization, BMSCs-seeded on Col-GAG and MC-GAG scaffolds in the presence and absence of BMP-2 $(50 \mathrm{ng} / \mathrm{mL})$ were subjected to histologic analysis at 6

A

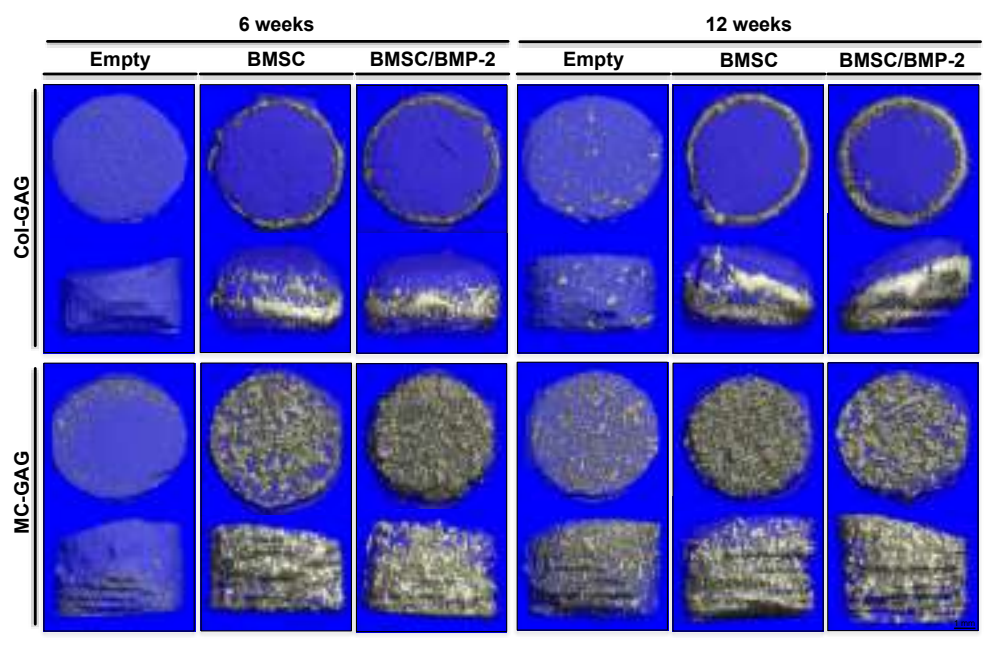

B

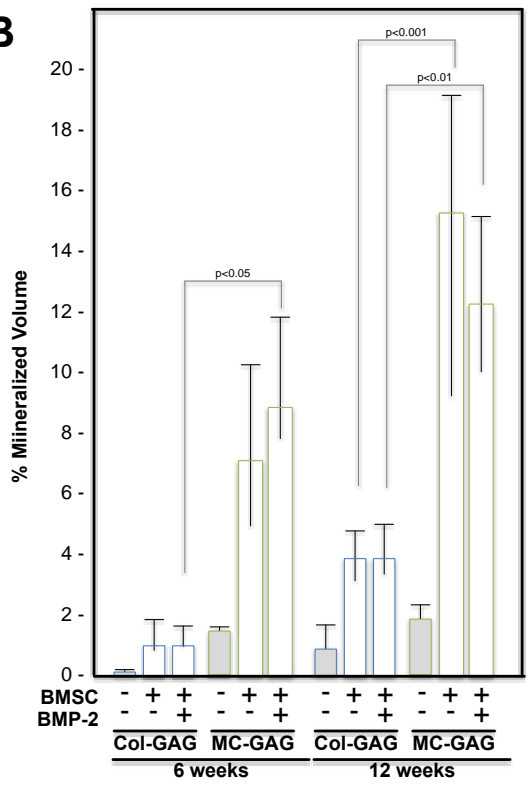

Figure 2. In vitro mineralization on $\mu \mathrm{CT}$ scanning of BMSCs cultured on Col-GAG and MC-GAG scaffolds in the absence and presence of BMP-2

(A) Representative 3D reconstructed $\mu \mathrm{CT}$ scans of histologic sections of collagen scaffolds cultured with BMSCs in osteogenic medium in the presence or absence of BMP-2 for 6 and 12 weeks on Col-GAG and MC-GAG scaffolds. Empty scaffolds at 6 and 12 weeks are shown for comparison. (B) Quantification of mineralized content on $\mu \mathrm{CT}$ scans in triplicate. 
and 8 weeks of culture (Figure 1D). In contrast to the Col-GAG scaffolds, MC-GAG scaffolds efficiently formed mineralized bone seen in the absence and presence of BMP2. At 8 weeks of culture, Col-GAG scaffolds demonstrated a minimal amount of mineralization largely at the surfaces of the scaffold.

$\mu \mathrm{CT}$ scanning was also performed on all scaffolds and their respective empty scaffold controls at 6 and 12 weeks of culture in osteogenic medium to evaluate in vitro mineralization (Figure 2). Similar to the histologic analyses, $\mu \mathrm{CT}$ scanning showed efficient mineralization of MC-GAG scaffolds, whereas non-mineralized Col-GAG scaffolds demonstrated only mineralization at the edges of the scaffold. When mineralized volume was quantified as a percentage of the total volume of the scaffold (Figure 2B), there was a significant effect of type of scaffold on percent mineralized volume $[F(11,18)=16.635, p<0.001]$. Post hoc comparisons using the Tukey criterion indicated that, at six weeks, the mineralization achieved in BMSCs cultured on MC-GAG was greater than Col-GAG in the presence of BMP-2 $(p<0.05)$. However, at 12 weeks of culture, MC-GAG cultured with BMSCs in the absence or presence of BMP-2 demonstrated increased mineralization when compared to the respective Col-GAG scaffolds ( $p<0.001$ and $p<0.01$, respectively). Both untreated and BMP-2 treated MCGAG/BMSCs scaffolds demonstrated significantly greater mineralization when compared to empty MC-GAG scaffolds at 12 weeks ( $p<0.001$ and $p<0.01$, respectively). In addition, treatment with BMP-2 did not significantly increase or decrease mineralization. Taken together, expression of osteogenic genes such as ALP and Col I were increased by BMP-2 treatment in either scaffold early during osteogenesis. However, as osteogenesis progressed, neither $\mathrm{OCN}$ expression nor mineralization was 
significantly influenced by BMP-2 treatment in MC-GAG scaffolds. Rather, only the type of scaffold influenced the quantity of mineralization.

\section{BMPs are differentially expressed and Smad1/5 is constitutively activated in}

\section{BMSCs on MC-GAG scaffolds.}
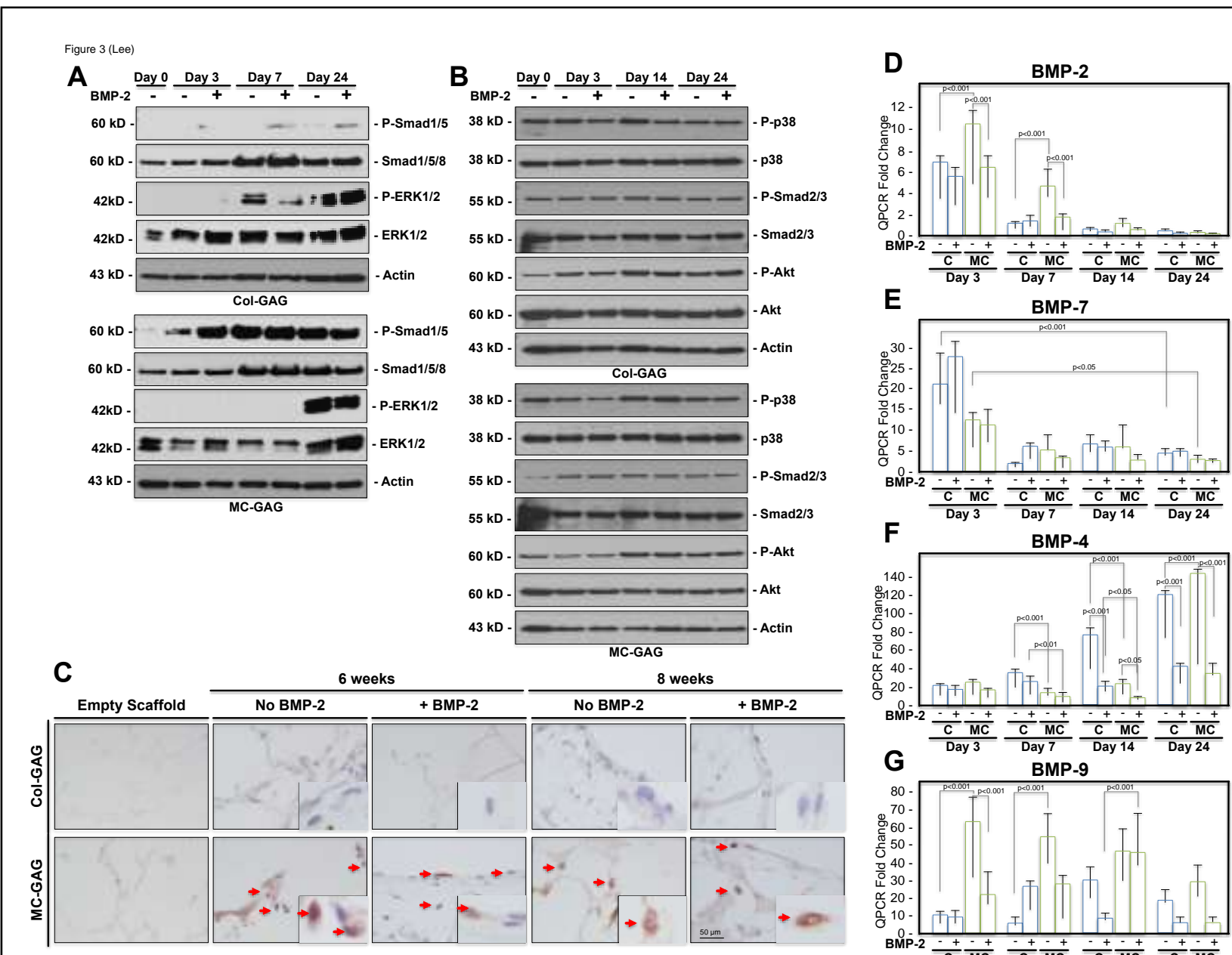

E

BMP-7
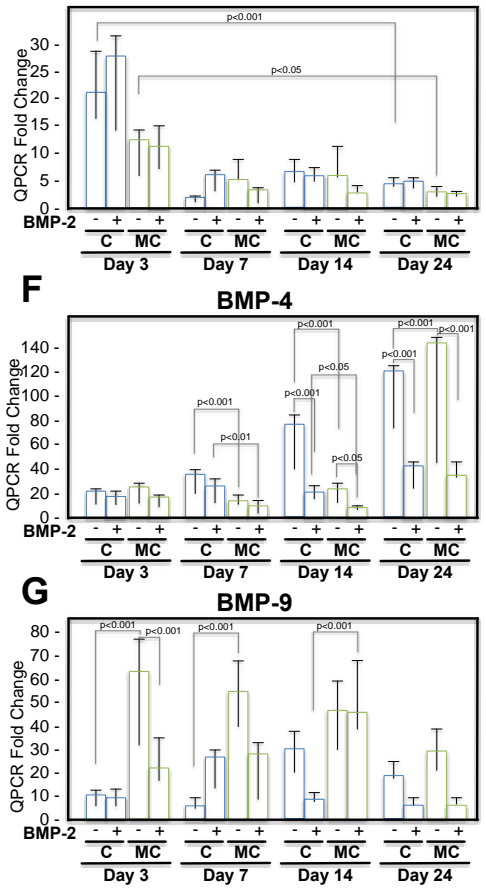

Figure 3. in vitro BMP expression and activation of intracellular mediators of BMP receptor signalling by BMSCs cultured on Col-GAG and MC-GAG

(A) Western blot of phosphorylated Smad 1/5 (P-Smad1/5), total Smad (Smad 1/5/8), phosphorylated ERK1/2 (P-ERK1/2), total ERK1/2, and actin controls. (B) Western blot of phosphorylated p38 (P-p38), total p38, phosphorylated Smad 2/3 (P-Smad2/3), total Smad 2/3, phosphorylated Akt (P-Akt), total Akt, and actin controls. (C) BMSCs cultured on Col-GAG and MC-GAG scaffolds were stained with anti-P-Smad1/5 at 6 and 8 weeks in the absence and presence of BMP-2. P-Smad1/5 is minimally found in BMSCs on Col-GAG scaffolds. PSmad1/5 strongly stains the nucleus in BMSCsf cultured on MC-GAG scaffolds with minimal to no increase in response to BMP-2. High magnification inserts are shown. Arrows denote nuclei. Real-time RT-PCR analysis of (D) BMP-2, (E) BMP-7, (F) BMP-4, and (G) BMP-9 expression in BMSCs on MC-GAG and Col-GAG scaffolds with and without BMP-2 $(50 \mathrm{ng} / \mathrm{mL})$. Data is expressed as the mean $\pm S D$ of three independent experiments in relation to day 0 values. $P$ values are shown in the figure. 
To elucidate the mechanism behind increased osteogenesis in MC-GAG scaffolds in relationship to Col-GAG scaffolds, the canonical (Smad dependent) and non-canonical (Smad independent) pathways of BMP receptor signaling were investigated (Figure 3A-B). In Col-GAG scaffolds, p-Smad1/5 was demonstrated only in response to BMP-2 stimulation on day 3 and day 7 (Figure $3 A$ ). On day 24, a small amount of $p$-Smad1/5 was detected in the untreated Col-GAG scaffolds that was slightly increased with BMP-2. MC-GAG scaffolds were dramatically different in both the quantity of Smad1/5 phosphorylation and response to BMP-2. Even at day 0, MC-GAG scaffolds showed a weak presence of $p-S m a d 1 / 5$. P-Smad1/5 was increased at day 3 , 7, and 24 in both BMP-2 untreated and treated scaffolds. Interestingly, BMP-2 had a stimulatory effect in MC-GAG scaffolds at day 3, but this difference was no longer present at the longer timepoints.

In contrast to Smad phosphorylation, p-ERK1/2 was elevated in the Col-GAG scaffolds in comparison to the MC-GAG scaffolds (Figure 3A). At day 7, BMP-2 stimulation resulted in a slight reduction in p-ERK1/2 in Col-GAG scaffolds. At day 24, both untreated and treated Col-GAG scaffolds showed elevated levels of $p-E R K 1 / 2$. In contrast, p-ERK1/2 was not seen in the MC-GAG scaffolds until day 24. Similar to ColGAG scaffolds, BMP-2 appeared to slightly decrease the amount of p-ERK1/2 at day 24 in MC-GAG scaffolds.

To determine whether other noncanonical downstream signaling molecules were affected, p38, Smad 2/3, and Akt were investigated (Figure 3B). Unlike Smad1/5 and ERK1/2, there were no differences detected between the two scaffolds types or induction by BMP-2 addition. These data show that MC-GAG scaffolds upregulate the BMPR canonical receptor Smad1/5 phosphorylation in BMSCs. This effect can be 
augmented at early timepoints (day 3) with exogenous BMP-2. However, at later timepoints (day 7 and 24), Smad 1/5 phosphorylation in MC-GAG scaffolds occurred even in the absence of exogenous BMP-2. Within the BMPR non-canonical signaling pathways, MC-GAG negatively regulated ERK1/2 phosphorylation early in osteogenic differentiation.

Following phosphorylation, Smad1/5 translocates to the nucleus to activate transcription of downstream targets. To confirm that phosphorylated Smad1/5 detected in Western blots corresponded to nuclear translocation, Col-GAG and MC-GAG scaffolds seeded with BMSCs at 6 and 8 weeks of culture, untreated and treated with BMP-2 stimulation, were subjected to immunohistochemistry with an antibody against $p$ Smad1/5 (Figure 3C). Strong staining and nuclear localization of $p$-Smad1/5 was seen in the presence and absence of BMP-2 in MC-GAG scaffolds at all timepoints. Col-GAG showed minimal staining of $p-S m a d 1 / 5$ with few cells demonstrating nuclear localization.

In combination, these data suggested that autogenous activation of the BMP receptor signaling pathway in BMSCs was stimulated by MC-GAG scaffolds. To further elucidate whether this activation was due to specific osteogenic BMP production, quantitative RT-PCR was performed for BMP-2, -4, -7, and -9 (Figure 3D-G). A significant effect of type, timing, and BMP-2 treatment of scaffold on gene expression was found for BMP-2 $[F(15,32)=69.736, p<0.001]$, BMP-4 $[F(15,32)=238.264$, $p<0.001]$, BMP-7 $[F(15,32)=18.488, p<0.001]$, and BMP-9 $[F(15,32)=12.131, p<0.001]$. Production of BMP-2 mRNA was found to be higher in MC-GAG scaffolds in comparison to Col-GAG scaffolds at the day $3(p<0.001)$ and $7(p<0.001)$ timepoints. BMP-2 expression was highest early and decreased with time in culture $(p<0.001)$. In MC-GAG scaffolds, exogenous BMP-2 negatively regulated BMP-2 expression at days 3 
$(p<0.001)$ and $7(p<0.001)$. BMP-7 also displayed an initial increase in expression at the day 3 timepoint, followed by decreased expression thereafter in either Col-GAG or MCGAG scaffolds. Although there appeared to be slightly more BMP-7 expression in ColGAG versus MC-GAG, this did not reach statistical significance. Unlike BMP-2 expression, exogenous BMP-2 did not have any significant impact on BMP-7 expression. BMP-4 expression differed in comparison to the expression patterns of BMP-2 and BMP-7. Expression of BMP-4 in Col-GAG scaffolds was significantly higher than MCGAG scaffolds on days 7 and 14. However, MC-GAG scaffolds surpassed Col-GAG scaffolds in BMP-4 expression at the day 24 timepoint suggesting a delay in BMP-4 expression on MC-GAG. Expression of BMP-4 was significantly downregulated with exogenous BMP-2 in either Col-GAG or MC-GAG. Interestingly, BMP-9 mRNA was also found to be much higher in MC-GAG scaffolds in comparison to Col-GAG scaffolds by approximately 6 fold in the day $3(p<0.001)$ and $7(p<0.001)$ timepoints. BMP-9 expression decreased with time in MC-GAG scaffolds while BMP-9 expression did not demonstrate statistically significant differences over time in Co-GAG scaffolds. Similar to BMP-2, BMP-9 expression was initially downregulated by exogenous BMP-2 in MCGAG scaffolds. However, this effect diminished with time. Taken together, efficient osteogenic differentiation of primary rabbit BMSCs on MC-GAG scaffolds correlated with activation of the canonical BMP receptor signaling pathway driven by transcriptional activation of BMP-2 and -9 early and BMP-4 late during differentiation. 
Figure 4 (Lee)
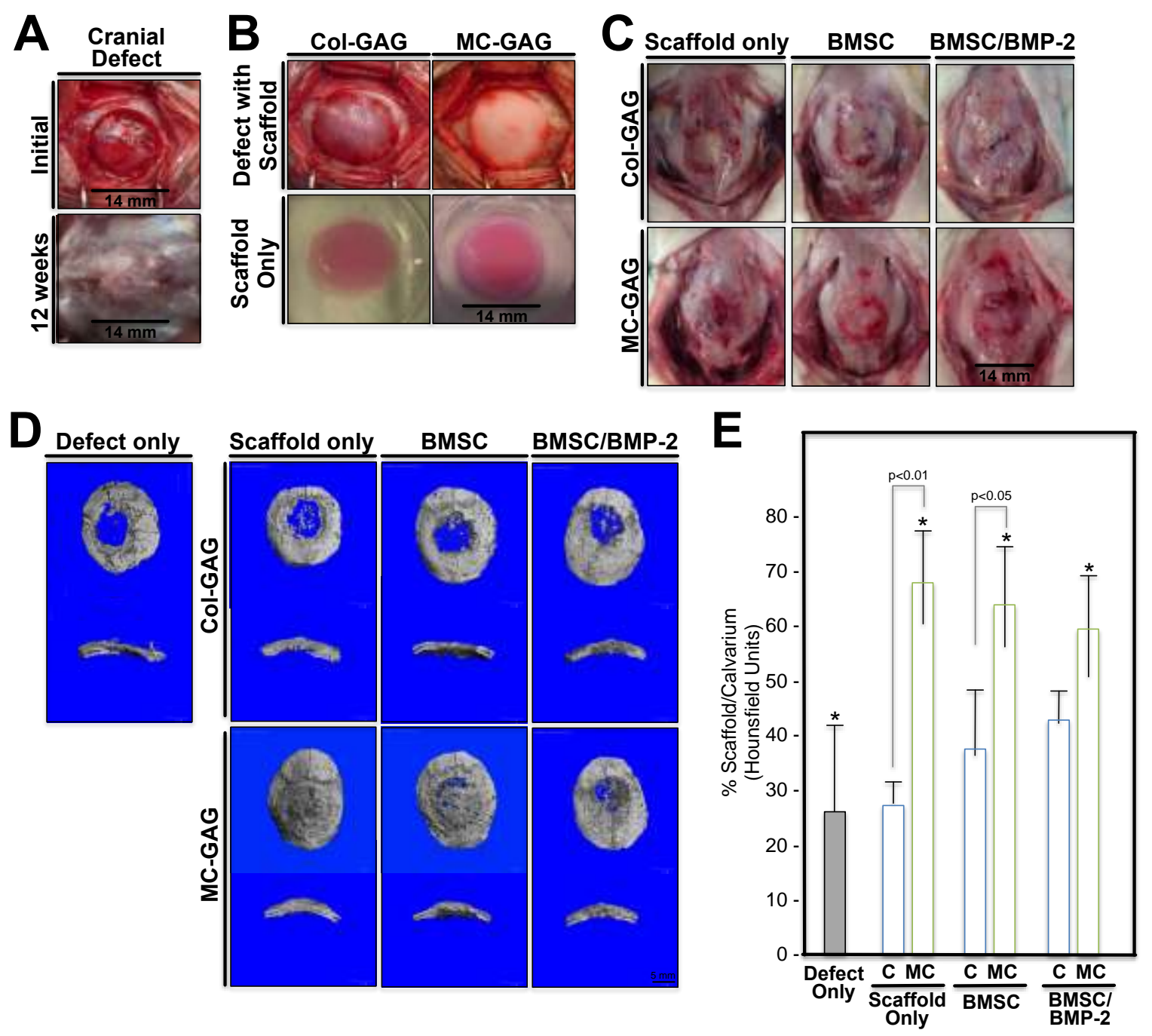

Figure 4. In vivo bone healing in critical sized rabbit cranial defects implanted with Col-GAG and MC-GAG scaffolds

(A) $14 \mathrm{~mm}$ in diameter cranial defects were surgically created in the parietal bones using a hand powered trephine. Initial defect prior to implantation (top) and 12 weeks post-implantation (bottom) cranial defects are shown. (B) Col-GAG and MC-GAG scaffolds at the time of implantation (top) and in ex vivo cultures (bottom). (C) 12 weeks following implantation, the rabbit skull containing the respective implants were grossly examined and explanted. Scaffold only denotes Col-GAG or MCGAG scaffolds without cells or BMP-2, BMSC denotes scaffolds cultured only with BMSCs, and $B M S C / B M P-2$ denotes scaffolds cultured with BMSCs and rhBMP-2 prior to implantation. (D and E) microCT scanning of explanted rabbit skulls. Defect only denotes cranial defect that did not receive reconstruction with any scaffolds. (D) Representative three-dimensional reconstructions of the CT scans with cross sections are shown. (E) Ratio of densities between the defect and the native calvarium (Hounsfield units) were calculated from a cylindrical volume encompassing the defect and internally controlled by the density of native calvarium. P values are shown, ${ }^{*}$ denotes $p<005$. C, Col-GAG; MC, MC-GAG; Defect only, cranial defect without reconstruction; Scaffold only, defects reconstructed with scaffolds without BMSCs or BMP-2; BMSC, defects reconstructed with scaffolds precultured with BMSCs without BMP-2; BMSC/BMP-2, defects reconstructed with scaffolds precultured with BMSCs and rhBMP-2. 


\section{Critical sized rabbit cranial defects are regenerated with nanoparticulate mineralized collagen glycosaminoglycan scaffolds in the absence of ex vivo progenitor cells expansion or exogenous BMP-2.}

With the establishment that rabbit primary BMSCs demonstrated similar mineralization and signalling mechanisms to hMSCs, we utilized a rabbit cranial defect model to compare the differences in in vivo bone healing in Col-GAG and MC-GAG scaffolds untreated or treated with BMP-2 (Figure 4). Primary BMSCs were first isolated from 6-8 week old rabbits and cultured ex vivo on Col-GAG or MC-GAG with and without BMP-2. After one week of culture, the scaffolds were implanted in critical-sized rabbit calvarial defects $14 \mathrm{~mm}$ in diameter and bone healing was evaluated after 12 weeks (Figure 4C).

The explanted skulls included native calvarium containing unreconstructed defects (Defect only), defects reconstructed with empty scaffolds (Scaffold only), defects reconstructed with scaffolds loaded with BMSCs, or defects reconstructed with scaffolds loaded with BMSCs and treated with BMP-2 (BMSC/BMP-2). Scaffolds were subjected to micro-CT scanning to assess mineralized content (Figure 4D-E). Quantification of the amount of mineralized content was derived by the average density in Hounsfield units from the micro-CT scan within a fixed volume of the defect and within the native calvarium. This was expressed as a ratio of defect to calvarium density. There was a significant effect of type of defect reconstruction on density $[F(6,18)=11.255$, $\mathrm{p}<0.001]$. Post hoc comparisons using the Tukey criterion indicated that the mean density achieved in unreconstructed defects $(26.5 \pm 15.6 \%)$ was significantly lower than defects reconstructed with empty MC-GAG scaffolds $(68.5 \pm 4.4 \%, p<0.001)$, MCGAG/BMSCs $(64.6 \pm 10.2 \%, p<0.01)$, or MC-GAG/BMSCs/BMP-2 $(59.8 \pm 0.09 \%$, 

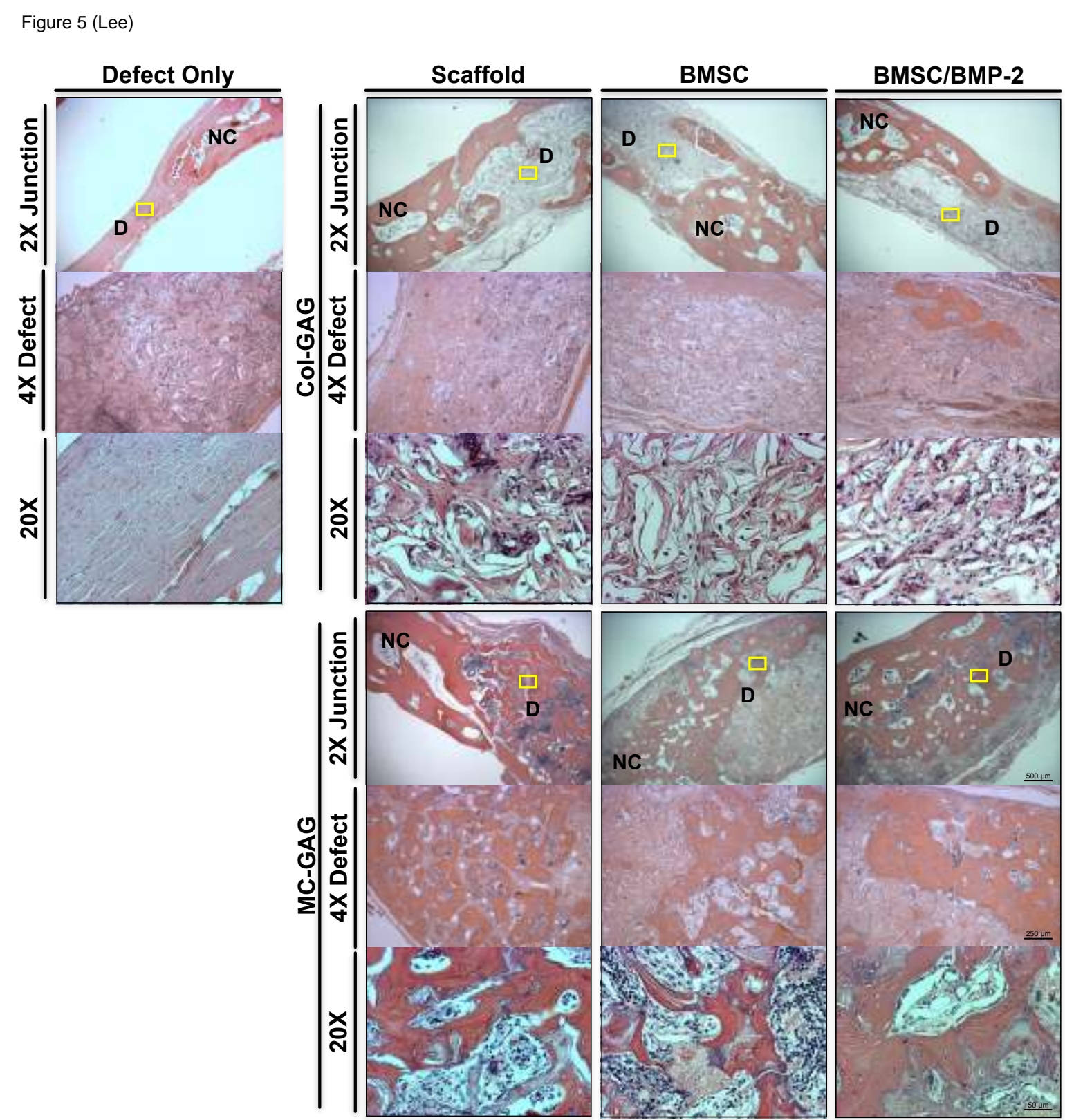

Figure 5. Histologic analyses of in vivo bone healing in critical sized rabbit cranial defects implanted with Col-GAG and MC-GAG scaffolds

H\&E staining of histologic sections of Col-GAG and MC-GAG scaffolds within rabbit cranial defects explanted after 12 weeks. Unreconstructed defect (Defect only) is depicted to the left and reconstructed defects are depicted to the right. Views in various magnification at different areas of the explanted skulls are shown. The junctions between the defect and native calvarium are depicted at low magnification ( $2 X$ Junction). The central areas of the defect are depicted at low magnification (4X Defect). Yellow rectangles on $2 X$ Junction panels indicate area corresponding to each high magnification view (20X). NC, normal calvarium; D, defect; Defect only, cranial defect without reconstruction; Scaffold, defects reconstructed with scaffolds without BMSCs or BMP-2; BMSC, defects reconstructed with scaffolds precultured with BMSCs without BMP-2; BMSC/BMP-2, defects reconstructed with scaffolds precultured with BMSCs and BMP-2.

$\mathrm{p}<0.01)$. Differences in densities between unreconstructed defects and Col-GAG 
reconstructed defects did not reach statistical significance. When comparing Col-GAG and MC-GAG, empty MC-GAG defects produced more mineralized volume than empty Col-GAG $(p<0.01)$ and MC-GAG/BMSCs produced more mineralized volume than ColGAG/BMSCs $(p<0.05)$. However, differences in density between MCGAG/BMSCs/BMP-2 versus Col-GAG/BMSCs/BMP-2 did not reach statistical significance. Lastly, no significant differences in density could be demonstrated between any of the MC-GAG scaffolds suggesting that neither addition of BMSCs or BMSCs/BMP-2 contributed additional bone healing.

Histologic analyses of explanted cranial defects were also performed to confirm the presence of mineralization (Figure 5). Novel bone, as noted by a decreased thickness in comparison to native calvarium as well as a less organized appearance, was identified at the junction between the scaffold and the native bone in all specimens. In the unreconstructed and Col-GAG reconstructed defects, minimal mineralized content was found in the central portions of the defect whereas the central portions of MC-GAG scaffolds contained significantly more mineralized bone. Thus, the histologic and microCT analyses of critical-sized rabbit cranial defects suggest that MC-GAG scaffolds regenerate more bone than Col-GAG scaffolds independent of pre-culturing with BMSCs or BMSCs/BMP-2. 

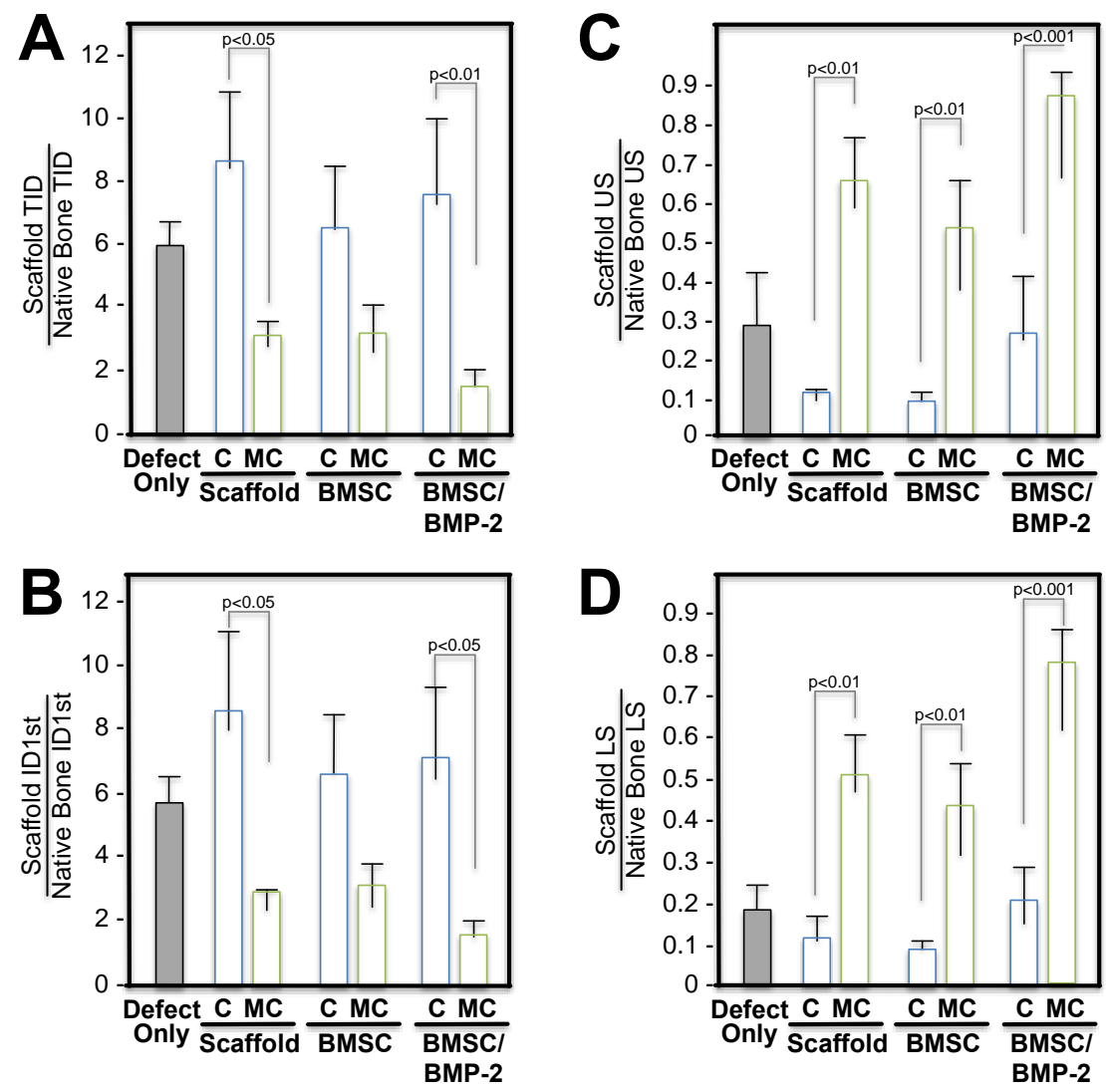

Figure 6. Biomechanical strength and stiffness of in vivo regenerated bone within Col-GAG and MC-GAG implanted cranial defects

Reference point indentation was performed in five areas of the cranial defects implanted with scaffolds and the native calvarium to evaluate biomechanical strength and stiffness. The strength, or resistance to fracture, was measured using the total indentation distance (TID) and first cycle indentation distance (ID1st) in $\mu \mathrm{m}$. The stiffness was measured using the loading slope (LS) and unloading slope (US). Strength and stiffness measurements were expressed as a ratio between defect and native calvarium. C, Col-GAG; MC, MCGAG; Defect only, cranial defect without reconstruction; Scaffold, defect reconstructed with scaffolds without BMSCs or BMP-2; BMSC, defect reconstructed with scaffolds precultured with BMSCs without BMP-2; BMSC/BMP-2, defect reconstructed with scaffolds precultured with BMSCs and rhBMP-2.

Type of defect reconstruction was evaluated with biomechanical testing via reference point indentation on new bone formation (Figure 6). Following explantation, the rabbit skulls were subjected to 40 rounds of bioindentation in the defect as well as the native calvarium. All bioindentation measurements were expressed as a ratio between defect to native bone in order to internally control for differences in bone healing and bone thickness between animals. Similar to previous reports, total 
indentation distance (TID) and first cycle indentation distances (ID 1st) were used as measures of microfracture resistance, whereas the unloading slope (US) and loading slope (LS) were used as measures of stiffness and elastic modulus [36, 37]. Differences in $\operatorname{TID}[F(6,12)=7.693, p=0.001]$, ID1st $[F(6,12)=8.318, p=0.001]$, US $[F(6,12)=$ 21.787, $p<0.001]$, and $\operatorname{LS}[F(6,12)=34.719, p<0.001]$ were found to be significant between cranial defects reconstructed with Col-GAG versus MC-GAG scaffold (Figure 6A-D). Post hoc comparisons using the Tukey criterion indicated that TID was decreased in defects reconstructed with empty MC-GAG $(p<0.05)$ and MCGAG/BMSC/BMP-2 $(p<0.01)$ when compared to the respected Col-GAG counterparts. When comparing the first cycle indentation distance, an indicator of density and mineralization, both empty MC-GAG $(p<0.05)$ and MC-GAG/BMSC/BMP-2 $(p<0.05)$ were reduced in comparison to the respective Col-GAG counterparts. When measures of stiffness were compared, both US and LS demonstrated increased values of each MC-GAG reconstructed defect in comparison to the corresponding Col-GAG defect. However, differences between the MC-GAG scaffolds were also seen where MCGAG/BMSC/BMP-2 demonstrated increased stiffness when compared to defects reconstructed with empty MC-GAG or MC-GAG/BMSC. Derived from loading and unloading slopes, the stiffness of MC-GAG reached between $50-80 \%$ of native bone. 


\section{Discussion}

Approaches to developing clinically translatable skeletal regenerative technologies have depended upon synthetic scaffolds, progenitor cells, and growth factors. However, progress in bone engineering have been impeded by the lack of optimized biomimetic and osteogenic reagents, the dependency on ex vivo cultures of large quantities of stem cells, and the usage of supraphysiologic dosages of growth factors thereby resulting in various unintended consequences [19-21]. Our current work investigated the healing of rabbit cranial defects with a custom nanoparticulate mineralized collagen glycosaminoglycan scaffold and detailed the contribution of ex vivo pre-culturing with bone marrow stromal cells and exogenous BMP-2. Our data showed that MC-GAG supported in vivo bone healing better than non-mineralized collagen glycosaminoglycan scaffolds (Col-GAG) and that this was possible even without ex vivo cultures with BMSCs or addition of exogenous growth factor. At the mechanistic level, we demonstrated that improved osteogenesis on MC-GAG scaffolds corresponded to differential temporal transcription of BMPs (BMP-2 and -9 early, BMP-4 late), thereby inducing autogenous BMP receptor signaling via phosphorylation of Smad 1/5. Although the in vivo regenerated bone was not identical in thickness to native bone, scaffold directed healing reached $>60 \%$ of the density of native calvarium at 3 months (Figure 4E and 5). From our biomechanical studies, the strength was less than native bone (Figure 6A-B) and stiffness was $50-80 \%$ of native bone (Figure $6 \mathrm{C}-\mathrm{D}$ ). However, given that the function of the calvarium is non-weight bearing, the differences in biomechanical resistance to fracture and stiffness from native bone are not significant for this particular clinical application. Previous work by our groups have demonstrated bioinspired methods for increasing the tensile mechanical strength of collagen 
biomaterials independent from the scaffold architecture [38, 39]. Ongoing efforts are extending these design concepts to improve the compressive strength of these mineralized constructs to support application in a wider range of clinical applications.

Our findings point out a common theme and reasoning for using progenitor cells. In most circumstances, the efficiency of in vivo bone healing is low in the absence of progenitor cells, as seen in Col-GAG scaffolds. Progenitor cells and growth factors, thus, augment osteogenesis by directing the process after implantation. However, the core problem is not a need for stem cells or growth factors, but an inability to optimize conditions with endogenous materials for adequate regeneration. Given that MC-GAG has the ability to demonstrate bone healing in the absence of ex vivo cultures with BMSCs, it is likely that qualities of the scaffold recruit osteoprogenitors and stimulate differentiation to bone. Other investigators have reported using cell-free scaffold implantation for regeneration, however the quantity of bone formed is less than our current report and the mechanism responsible for regeneration was not elucidated [40]. The next question is, thus, what is the source of such progenitor cells? Several investigators have demonstrated that both the dura mater and the cranial suture mesenchyme provide osteoprogenitors and serve to direct physiological calvarial suture fusion [41, 42]. In our current work, we have noted infiltration of cellular material through the entirety of the scaffold even without ex vivo BMSC preculturing. In addition, we have also noted that bone formation occurs at the periphery as well as centrally suggestive that cellular infiltration was not localized to the peripheral bone. Thus, it is likely that local osteoprogenitors from both the surrounding bone and dura mater are responsible for the mineralized content in implanted empty MC-GAG scaffolds.

There are several unanswered questions relating to the intersection between in 
vitro and in vivo studies. Components of the osteogenic differentiation medium used in vitro, such as glucocorticoids, are likely contributors to BMSC osteogenesis. However, the combination of glucocorticoids and MC-GAG scaffolds on BMSCs is unknown. Additionally, cell seeding of scaffolds for in vitro versus in vivo experiments were different due to the differences in the size of the scaffolds. Differences in cell number or cell density in in vivo bone healing may contribute to differentiation, mineralization, or BMP signalling. Lastly, all of the animals used in this study were female and, thus, the potential contributions of estrogen in bone healing cannot be excluded. Although all of the former considerations are held as constants throughout the study to formulate the current conclusions, further study in understanding interactions of the MC-GAG scaffold on BMSCs may be warranted.

Addition of exogenous BMP-2 resulted in a downregulation of BMP-2, -9 , and -4 expression, whereas differential temporal expression of all three BMPs was seen in the MC-GAG scaffolds that were left untreated. This concept of temporal regulation of differential BMP expression has been suggested by others and may be a part of normal physiological osteogenic differentiation [43]. This may be indicative of a mechanistic reason for dysregulated bone formation reported during clinical BMP-2 usage. These data point out a significant downfall in depending on supraphysiologic doses of growth factors for osteogenic stimulation. In addition, the ability for biomimetic materials to stimulate efficient osteogenesis provides an alternate, potentially more controlled, pathway for skeletal regeneration.

Downstream BMP receptor signaling was activated via the canonical Smad1/5 pathway, suggesting that MC-GAG scaffolds activate BMPR signaling via autogenous ligand expression. However, the inductive agent for BMP expression remains to be 
elucidated. Although other investigators have observed osteogenic properties from organic components of the extracellular matrix [44, 45], Col-GAG and MC-GAG scaffolds are composed of comparable organic components. Thus, the inorganic component differentiates between the two scaffolds. One of the most recent developments in osteogenic signaling relates to the contributions of inorganic ions such as phosphate to induction of osteogenesis [46, 47]. Clinically, both hyperphosphatemia and increased serum concentrations of BMP-9 are present in renal failure patients and have been demonstrated to synergistically induce osteogenic differentiation of vascular smooth muscle cells [48]. BMP-9 is interesting in that it has been reported to be the most osteogenic member of all BMP molecules although relatively little is understood about its physiologic and pathologic functions [49]. This clinical situation is analogous to the microenvironment provided by the MC-GAG scaffold in that inorganic phosphate is concentrated and an increase in BMP-9 is detected in BMSCs undergoing osteogenic differentiation. Thus, the efficiency of osteogenesis of MC-GAG may be related to the nanoparticulate distribution of inorganic matter. Further understanding of inorganic ion concentrations in osteogenic signaling and defect healing will be an important concept for the design of future biomaterials.

Acknowledgements: This work was supported by the US Department of Veterans Affairs under award numbers IK2 BX002442-01A2 (JCL) and 1I01BX001367-01A2 (TAM), the Aramont Foundation (TAM), and the Jean Perkins Foundation (JCL). Research reported in this publication was also supported by the National Institute of 
Arthritis and Musculoskeletal and Skin Diseases of the National Institutes of Health under award number R21 AR063331 (BACH). The content is solely the responsibility of the authors and does not necessarily represent the official views of the National Institutes of Health. Additional support was provided by project number S-14-54H by AO Foundation, Switzerland (BACH). DWW was funded at UIUC from National Science Foundation (NSF) Grant 0965918 IGERT: Training the Next Generation of Researchers in Cellular \& Molecular Mechanics and BioNanotechnology. 


\section{References}

[1] Ebraheim NA, Elgafy $\mathrm{H}, \mathrm{Xu}$ R. Bone-graft harvesting from iliac and fibular donor sites: techniques and complications. J Am Acad Orthop Surg. 2001;9:210-8.

[2] Arrington ED, Smith WJ, Chambers HG, Bucknell AL, Davino NA. Complications of iliac crest bone graft harvesting. Clin Orthop Relat Res. 1996:300-9.

[3] Ma D, Ren L, Chen F, Liu Y, Zhang J, Xue Z, et al. Reconstruction of rabbit criticalsize calvarial defects using autologous bone marrow stromal cell sheets. Annals of plastic surgery. 2010;65:259-65.

[4] Stocker G, Meyer HE, Wagener C, Greiling H. Purification and N-terminal amino acid sequence of a chondroitin sulphate/dermatan sulphate proteoglycan isolated from intima/media preparations of human aorta. The Biochemical journal. 1991;274 ( Pt 2):415-20.

[5] Lee JC, Kleiber GM, Pelletier AT, Reid RR, Gottlieb LJ. Autologous immediate cranioplasty with vascularized bone in high-risk composite cranial defects. Plast Reconstr Surg. 2013;132:967-75.

[6] Szpalski C, Wetterau M, Barr J, Warren SM. Bone tissue engineering: current strategies and techniques--part I: Scaffolds. Tissue Eng Part B Rev. 2012;18:246-57. [7] Szpalski C, Sagebin F, Barbaro M, Warren SM. The influence of environmental factors on bone tissue engineering. J Biomed Mater Res B Appl Biomater. 2012. [8] Ducy P, Karsenty G. The family of bone morphogenetic proteins. Kidney Int. 2000;57:2207-14.

[9] Miyazono K, Kamiya Y, Morikawa M. Bone morphogenetic protein receptors and signal transduction. J Biochem. 2010;147:35-51. 
[10] Kretzschmar M, Doody J, Massagué J. Opposing BMP and EGF signalling pathways converge on the TGF-beta family mediator Smad1. Nature. 1997;389:618-22. [11] Mukherjee A, Wilson EM, Rotwein P. Selective signaling by Akt2 promotes bone morphogenetic protein 2-mediated osteoblast differentiation. Mol Cell Biol. 2010;30:1018-27.

[12] Li C, Yang X, He Y, Ye G, Li X, Zhang X, et al. Bone morphogenetic protein-9 induces osteogenic differentiation of rat dental follicle stem cells in P38 and ERK1/2 MAPK dependent manner. Int J Med Sci. 2012;9:862-71.

[13] Nolan K, Thompson TB. The DAN family: Modulators of TGF- $\beta$ signaling and beyond. Protein Sci. 2014;23:999-1012.

[14] Aspenberg P, Jeppsson C, Economides AN. The bone morphogenetic proteins antagonist Noggin inhibits membranous ossification. J Bone Miner Res. 2001;16:497500.

[15] Gazzerro E, Canalis E. Bone morphogenetic proteins and their antagonists. Rev Endocr Metab Disord. 2006;7:51-65.

[16] Friedlaender GE. OP-1 clinical studies. J Bone Joint Surg Am. 2001;83-A Suppl 1:S160-1.

[17] Friedlaender GE, Perry CR, Cole JD, Cook SD, Cierny G, Muschler GF, et al. Osteogenic protein-1 (bone morphogenetic protein-7) in the treatment of tibial nonunions. J Bone Joint Surg Am. 2001;83-A Suppl 1:S151-8.

[18] Khan SN, Lane JM. The use of recombinant human bone morphogenetic protein-2 (rhBMP-2) in orthopaedic applications. Expert Opin Biol Ther. 2004;4:741-8. 
[19] Lewandrowski KU, Nanson C, Calderon R. Vertebral osteolysis after posterior interbody lumbar fusion with recombinant human bone morphogenetic protein 2: a report of five cases. Spine J. 2007;7:609-14.

[20] Smoljanovic T, Bojanic I, Delimar D. Adverse effects of posterior lumbar interbody fusion using rhBMP-2. Eur Spine J. 2009;18:920-3; author reply 4.

[21] Yee KS, Nguyen PD, Andrews BT, Lee JC, Bradley JP. Abstract 4: Decreased Secondary Bone Grafting but Poorer Midface Growth after Primary Alveolar Cleft Repair with Gingivoperiosteoplasty and rhBMP-2. Plast Reconstr Surg. 2014;133:975. [22] Kruger EA, Im DD, Bischoff DS, Pereira CT, Huang W, Rudkin GH, et al. In vitro mineralization of human mesenchymal stem cells on three-dimensional type I collagen versus PLGA scaffolds: a comparative analysis. Plast Reconstr Surg. 2011;127:2301-11. [23] Bergsma EJ, Rozema FR, Bos RR, de Bruijn WC. Foreign body reactions to resorbable poly(L-lactide) bone plates and screws used for the fixation of unstable zygomatic fractures. J Oral Maxillofac Surg. 1993;51:666-70. [24] Jiang WW, Su SH, Eberhart RC, Tang L. Phagocyte responses to degradable polymers. J Biomed Mater Res A. 2007;82:492-7.

[25] Walsh WR, Vizesi F, Michael D, Auld J, Langdown A, Oliver R, et al. Beta-TCP bone graft substitutes in a bilateral rabbit tibial defect model. Biomaterials. 2008;29:26671.

[26] Ren X, Bischoff D, Weisgerber DW, Lewis MS, Tu V, Yamaguchi DT, et al. Osteogenesis on nanoparticulate mineralized collagen scaffolds via autogenous activation of the canonical BMP receptor signaling pathway. Biomaterials. 2015;50:10714. 
[27] Lee JC, Pereira CT, Ren X, Huang W, Bischoff D, Weisgerber DW, et al. Optimizing Collagen Scaffolds for Bone Engineering: Effects of Cross-linking and Mineral Content on Structural Contraction and Osteogenesis. J Craniofac Surg. 2015.

[28] Harley BA, Leung JH, Silva EC, Gibson LJ. Mechanical characterization of collagenglycosaminoglycan scaffolds. Acta Biomater. 2007;3:463-74.

[29] Olde Damink LH, Dijkstra PJ, van Luyn MJ, van Wachem PB, Nieuwenhuis P, Feijen J. Cross-linking of dermal sheep collagen using a water-soluble carbodiimide. Biomaterials. 1996;17:765-73.

[30] Harley BA, Lynn AK, Wissner-Gross Z, Bonfield W, Yannas IV, Gibson LJ. Design of a multiphase osteochondral scaffold II: fabrication of a mineralized collagen-GAG scaffold. J Biomed Mater Res A. 2010;92:1066-77.

[31] Weisgerber DW, Kelkhoff DO, Caliari SR, Harley BAC. The impact of discrete compartments of a multi-compartment collagen-GAG scaffold on overall construct biophysical properties. J Mech Behav Biomed Mater. 2013;28:26-36.

[32] Ashby ER, Rudkin GH, Ishida K, Miller TA. Evaluation of a novel osteogenic factor, bone cell stimulating substance, in a rabbit cranial defect model. Plast Reconstr Surg. 1996;98:420-6.

[33] Caliari SR, Harley BA. Structural and Biochemical Modification of a Collagen Scaffold to Selectively Enhance MSC Tenogenic, Chondrogenic, and Osteogenic Differentiation. Adv Healthc Mater. 2014.

[34] Weisgerber DW, Kelkhoff DO, Caliari SR, Harley BA. The impact of discrete compartments of a multi-compartment collagen-GAG scaffold on overall construct biophysical properties. J Mech Behav Biomed Mater. 2013;28:26-36. 
[35] Roostaeian J, Carlsen B, Simhaee D, Jarrahy R, Huang W, Ishida K, et al.

Characterization of growth and osteogenic differentiation of rabbit bone marrow stromal cells. J Surg Res. 2006;133:76-83.

[36] Gallant MA, Brown DM, Organ JM, Allen MR, Burr DB. Reference-point indentation correlates with bone toughness assessed using whole-bone traditional mechanical testing. Bone. 2013;53:301-5.

[37] Diez-Perez A, Güerri R, Nogues X, Cáceres E, Peña MJ, Mellibovsky L, et al. Microindentation for in vivo measurement of bone tissue mechanical properties in humans. J Bone Miner Res. 2010;25:1877-85.

[38] Caliari SR, Mozdzen LC, Armitage O, Oyen ML, Harley BA. Award Winner in the Young Investigator Category, 2014 Society for Biomaterials Annual Meeting and Exposition, Denver, Colorado, April 16-19, 2014: Periodically perforated core-shell collagen biomaterials balance cell infiltration, bioactivity, and mechanical properties. $\mathrm{J}$ Biomed Mater Res A. 2014;102:917-27. [39] Caliari SR, Ramirez MA, Harley BA. The development of collagen-GAG scaffoldmembrane composites for tendon tissue engineering. Biomaterials. 2011;32:8990-8. [40] Rentsch C, Rentsch B, Heinemann S, Bernhardt R, Bischoff B, Förster Y, et al. ECM inspired coating of embroidered 3D scaffolds enhances calvaria bone regeneration. Biomed Res Int. 2014;2014:217078.

[41] Opperman LA, Chhabra A, Nolen AA, Bao Y, Ogle RC. Dura mater maintains rat cranial sutures in vitro by regulating suture cell proliferation and collagen production. $J$ Craniofac Genet Dev Biol. 1998;18:150-8.

[42] Zhao H, Feng J, Ho TV, Grimes W, Urata M, Chai Y. The suture provides a niche for mesenchymal stem cells of craniofacial bones. Nat Cell Biol. 2015;17:386-96. 
[43] Twine NA, Chen L, Pang CN, Wilkins MR, Kassem M. Identification of differentiation-stage specific markers that define the ex vivo osteoblastic phenotype. Bone. 2014;67:23-32.

[44] Rammelt S, Schulze E, Witt M, Petsch E, Biewener A, Pompe W, et al. Collagen type I increases bone remodelling around hydroxyapatite implants in the rat tibia. Cells Tissues Organs. 2004;178:146-57.

[45] Manton KJ, Leong DF, Cool SM, Nurcombe V. Disruption of heparan and chondroitin sulfate signaling enhances mesenchymal stem cell-derived osteogenic differentiation via bone morphogenetic protein signaling pathways. Stem Cells. 2007;25:2845-54.

[46] Shih YR, Hwang Y, Phadke A, Kang H, Hwang NS, Caro EJ, et al. Calcium phosphate-bearing matrices induce osteogenic differentiation of stem cells through adenosine signaling. Proc Natl Acad Sci U S A. 2014;111:990-5.

[47] Cushnie EK, Ulery BD, Nelson SJ, Deng M, Sethuraman S, Doty SB, et al. Simple signaling molecules for inductive bone regenerative engineering. PLoS One. 2014;9:e101627.

[48] Zhu D, Mackenzie NC, Shanahan CM, Shroff RC, Farquharson C, MacRae VE. BMP-9 regulates the osteoblastic differentiation and calcification of vascular smooth muscle cells through an ALK1 mediated pathway. J Cell Mol Med. 2015;19:165-74. [49] Luu HH, Song WX, Luo X, Manning D, Luo J, Deng ZL, et al. Distinct roles of bone morphogenetic proteins in osteogenic differentiation of mesenchymal stem cells. $\mathrm{J}$ Orthop Res. 2007;25:665-77. 
Tables: Table1. Primer Sequences

\begin{tabular}{|c|c|c|}
\hline Genes & Oligonucleotide Sequence & Accession \\
\hline$\beta$-Actin sense & 5'-TCACCCACACTGTGCCCCATCTACGA-3' & $\begin{array}{l}\text { From } \\
\text { PMID:23290592 }\end{array}$ \\
\hline$\beta$-Actin antisense & 5'-CAGCGGAACCGCTCATTGCCAATGG-3' & \\
\hline ALP sense & 5'-TTGCGCACGTCATGGCCCTC-3' & $\begin{array}{l}\text { From PMID: } \\
22894817\end{array}$ \\
\hline ALP antisense & 5'-CCCCATTAGGGGGCGTCACAT-3' & \\
\hline Col I sense & 5'-TGCGACATGGACACTGGGGC-3' & $\begin{array}{l}\text { From PMID: } \\
22894817\end{array}$ \\
\hline Col I antisense & 5'-GAGCCTTCGCTGCCGTACTCG-3' & \\
\hline OPN sense & 5'-AGTCTGATGAGTCTGATGAAGTCAC-3' & NM_001082194 \\
\hline OPN antisense & 5'-GTGACTTTGGGTTTCCACGC-3' & \\
\hline OCN sense & 5'-GGCGCCAACTGATCGACGGG-3' & $\begin{array}{l}\text { From PMID: } \\
22894817\end{array}$ \\
\hline $\mathrm{OCN}$ antisense & 5'-CGGGTTGAGCTCGCACACCT-3' & \\
\hline BMP2 sense & 5'ACGACATCCTGAGCGAGTTC-3‘ & NM_001082650 \\
\hline BMP2 antisense & 5'-CAAGTCCAGCATGTAGGGGG-3' & \\
\hline BMP4 sense & 5'- CCACCACGAAGAACATCTGGA-3' & NM_001195723 \\
\hline BMP4 antisense & 5'-TGTTTATCCGGTGGAAGCCC-3' & \\
\hline BMP7 sense & 5'-CTTCATCGACCCGGACACAG-3' & EU004072 \\
\hline BMP7 antisense & 5'-GACGTGGTCCCTTTGGGTC-3' & \\
\hline BMP9 sense & 5'-CGGAAGGGAACCTGGTCATT-3' & XM_002723523 \\
\hline BMP9 antisense & 5'-GACACTTCCAGGGTCTCCCA-3' & \\
\hline
\end{tabular}

ALP, alkaline phosphatase; Col I, type I collagen; OPN, Osteopontin, OCN, osteocalcin and BMP, bone morphogenetic protein. 